\title{
DETERMINANTES SOCIALES DEL INTENTO DE SUICIDO EN JÓVENES COLOMBIANOS
}

Fernando Salcedo Mejia

ALZAK Foundation

Colombia

Nelson Rafael Alvis Zakzuk

ALZAK Foundation G. I. en Gestión Hospitalaria y Políticas de Salud Colombia

Marley Jerez Arias Fundación SER SOCIAL Colombia

Justo Paz Wilches Mutual SER EPS

Colombia

Maria Carrasquilla Sotomayor ALZAK Foundation Colombia 
Panorama Económico, Vol. 27 - No. 3 (Julio - Septiembre de 2019), pp. 689-700

Nelson Rafael Alvis Zakzuk

Fernando Salcedo Mejia

Marley Jerez Arias

Justo Paz Wilches

Maria Carrasquilla Sotomayor

\title{
Determinantes sociales del intento de suicidio en jóvenes colombianos
}

\section{Resumen}

El presente estudio estudia la prevalencia y analiza los factores que determinan los intentos de suicidio en jóvenes entre 10-24 años. Se trata de un estudio descriptivo-correlacional con una muestra de 10.677 jóvenes. Se describieron las características sociodemográficas y se realizó un modelo probit para estimar los factores que se asocian a los intentos de suicidio en la población joven estudiada. El $2 \%$ de la muestra reportó intentos de suicidio y el 70,8\% se presentó en mujeres. El nivel de escolaridad del joven y los padres, las ideaciones suicidas y el maltrato físico fueron importantes predictores de los intentos de suicidios en jóvenes de los Montes de María.

Palabras clave: Intento de suicidio, determinantes, jóvenes, modelo probit.

\section{Déterminants sociaux de la tentative de suicide chez les jeunes colombiens}

\section{Résumé:}

La présente étude étudie la prévalence et analyse les facteurs qui déterminent les tentatives de suicide chez les jeunes de 10 à 24 ans. Il s'agit d'une étude descriptive-corrélationnelle avec un échantillon de 10 677 jeunes. Les caractéristiques sociodémographiques ont été décrites et un modèle probit a été élaboré pour estimer les facteurs associés aux tentatives de suicide dans la population jeune étudiée. $2 \%$ de l'échantillon ont signalé des tentatives de suicide et $70,8 \%$ se sont présentés chez les femmes. Le niveau de scolarité des jeunes et des parents, les idées suicidaires et les violences physiques étaient d'importants prédicteurs de tentatives de suicide chez les jeunes des Montes de María.

Mots clés: Tentative de suicide, déterminants, jeunesse, modèle probit.

\section{Social determinants of the suicide attempt in colombian young people}

\begin{abstract}
The present study studies the prevalence and analyzes the factors that determine suicide attempts in young people between 10-24 years. This is a descriptive-correlational study with a sample of 10,677 young people. Sociodemographic characteristics were described and a probit model was made to estimate the factors associated with suicide attempts in the young population studied. $2 \%$ of the sample reported suicide attempts and $70.8 \%$ showed up in women. The level of schooling of the youth and parents, suicidal ideations and physical abuse were important predictors of suicide attempts in youth in the Montes de María.
\end{abstract}

Keywords: Suicide attempt, determinants, youth, probit model. 


\section{Determinantes sociales del intento de suicidio en jóvenes colombianos}

INFORMACIÓN DEL ARTíCULO

Recepción del artículo: 26/02/2019

Concepto de evaluación: 20/03/2019

Aceptación del artículo: 06/06/2019
Fernando Salcedo Mejia ALZAK Foundation, Colombia

Nelson Rafael Alvis Zakzuk ALZAK Foundation / G. I. en Gestión Hospitalaria y Políticas de Salud, Colombia

Marley Jerez Arias Fundación SER SOCIAL, Colombia Justo Paz Wilches Mutual SER EPS, Colombia

Maria Carrasquilla Sotomayor ALZAK Foundation, Colombia

\section{INTRODUCCIÓN}

El suicido es una importante causa de mortalidad a nivel mundial que causa cada año cerca de 800.000 muertes y es la segunda causa de defunciones en el grupo etario de 15 a 29 años ("OMS," 2015). El 75\% de todos los suicidios se produce en países de ingresos bajos y medios. Los métodos e instrumentos más comunes de suicidio en todo el mundo son la ingestión de plaguicidas, el ahorcamiento y las armas de fuego ("OMS," 2015).

El suicidio, se definió por primera vez por Emile Durkheim como "todo caso de muerte que resulte directa o indirectamente de un acto, positivo o negativo, realizado por la víctima misma, sabiendo ella que debía producir este resultado (Durkheim, 1995)". La Organización Mundial de la Salud (OMS) lo define como el acto deliberativo de quitarse la vida (OMS, 2014). El intento de suicidio por su parte, se define como aquel acto que lleva a la intención de quitarse la vida y no se consuma por alguna circunstancia, por lo que no tiene un desenlace fatal (Gutierez Ana, Contreras Carlos, 2011).

Con el fin de luchar contra este problema de salud pública, la OMS recomienda a los países tener una estrategia integral enfocada a identificar grupos vulnerables con riesgo de suicidio, y mejorar su acceso a los servicios. Para que esta estrategia sea efectiva debe 
incluir tres aspectos: 1) Reducir porcentualmente la tasa de suicidio de ese país. 2) Tener la estadística de intervenciones de prevención de suicidios. 3) Disminuir el número de casos hospitalizados por intento de suicidio (OMS, 2014).

En Colombia, se han realizado investigaciones que estiman factores relacionados con los intentos de suicidios. En Manizales, por ejemplo, se estudió el riesgo suicida y sus factores asociados, encontrando que el $80 \%$ de los participantes reportó al menos un evento estresante en su vida, y de éstos el $82,1 \%$ estaba relacionado con la muerte de un familiar cercano. De igual manera, se relacionó la intimidación escolar (Bullying), la disfuncionalidad familiar, la baja autoestima, la depresión y el consumo de alcohol como factores de riesgo de los intentos de suicidio. (Diana Carolina Aguirre-Florez, Jose Jaime Castaño-Castrillon, 2013). Otro estudio realizado en Bogotá, el cual caracterizó pacientes que intentaron suicidarse, reveló que el 66,7\% fueron mujeres, 24,4\% estudiantes, 20,5\% desempleados y el $49 \%$ consumió alcohol previo al intento suicida. La no resolución de conflictos y la disfunción familiar fueron predictores de la persistencia del intento suicida (Pérez Isabel, 2004). De otra parte, otra investigación buscó describir y analizar el perfil epidemiológico y los determinantes del intento de suicidio en Colombia, revelando que los intentos de suicidio para el periodo de estudio estuvieron asociados con depresión grave y moderada, niveles moderados y graves de ansiedad y además se presentaron principalmente en edades entre 16 a 21 años (Gómez-Restrepo, Carlos. Rodriguez Malagón, Nelcy. Bohórquez P, 2002).

El instituto Nacional de Salud (INS) de Colombia, reportó que en 2015 se notificaron al Sistema Nacional de Vigilancia en Salud pública (Sivigila) 14.477 casos de intentos de suicidios por intoxicaciones, de las cuales el 53,5\% fueron intoxicaciones por medicamentos y $34,4 \%$ por plaguicidas. El 50,4\% de los casos reportados pertenecen a jóvenes entre los 15 y 19 años (30,7\%) y entre los 20 y 24 años (19,7 \%) (“INS," 2015).

Teniendo en cuenta lo anterior, surge la necesidad de estudiar la prevalencia de los intentos de suicidio y analizar los factores que determinan los intentos de suicidio en jóvenes colombianos entre 10-24 años de edad, especialmente en una subregión donde nunca se ha estudiado este problema.

\section{MÉTODO}

El presente es un estudio descriptivo-correlacional de corte transversal que describió la prevalencia y analizó los determinantes de los intentos de suicidio de jóvenes vulnerables de siete municipios del Caribe colombiano.

\section{Participantes}

Este estudio utilizó como principal fuente de información una encuesta diseñada, financiada y aplicada por la Fundación Ser Social. La población objetivo fueron jóvenes entre 10-24 años afiliados a una Empresa Promotora de Salud del régimen subsidiado (Mutual SER EPS) y residentes en siete municipios de Bolívar (El Guamo, Calamar, Zambrano, San Juan, San Jacinto y el Carmen de Bolívar) y el departamento de Sucre (San Onofre), pertenecientes a la subregión de los Montes de María. Para la recolección de los datos la Fundación Ser Social, aplicó de forma auto-administrada un cuestionario semiestructurado llamado "Escala de caracterización ser Joven" que indagó acerca de 
las características sociodemográficas, la salud sexual, mental y reproductiva de la población joven estudiada. Mediante muestreo aleatorio simple se seleccionó una muestra de 10.677 jóvenes. El trabajo de campo se realizó entre los meses de junio y diciembre de 2014.

\section{Modelación de los determinantes y análisis de la información}

Se describieron las frecuencias absolutas y relativas de los intentos de suicidio en adolescentes de los Montes de María, por municipio, grupo de edad, sexo y nivel de escolaridad.

Para estimar los determinantes de los intentos de suicidio, se utilizó un modelo Probit multivariado que tiene la siguiente forma funcional:

$$
D_{i}=\left\{\begin{array}{c}
1 \text { si decide la alternativa } 1\left(u_{1 i}>0\right) \\
0 \text { de lo contrario }
\end{array}\right\}
$$

La regla de decisión es maximizar la utilidad $\left(\mathrm{u}_{1}\right)$ :

$$
\begin{gathered}
u_{1 i}=X_{1 i} \beta+\varepsilon_{1 i} \\
u_{0 i}=0
\end{gathered}
$$

Supuesto: Los errores del modelo siguen una distribución normal $\varepsilon_{1} \sim N\left(0, \sigma^{2}\right)$.

$$
\begin{array}{lrl}
D_{1 i}=1 & \text { si } u_{1 i}=X_{1 i} \beta+\varepsilon_{1 i}>0 \\
D_{1 i}=0 & \text { de lo contrario }
\end{array}
$$

Debido a que el modelo Probit es un modelo de variable dependiente limitada, la estimación de los parámetros se realiza mediante el método de máxima verosimilitud, el cual sugiere que se elijan como estimados los valores de los parámetros que maximicen el logaritmo de la función de verosimilitud, como se expresa a continuación:

$$
\operatorname{Ln} L\left(\sigma^{2}, \beta \mid X\right)=\sum_{i=1}^{N}\left\{D_{i} \operatorname{Ln} \operatorname{Pr}\left(D_{i}=1 \mid X, \sigma^{2}, \beta\right)+\left(1-D_{i}\right) \operatorname{Ln}\left[1-\operatorname{Pr}\left(D_{i}=1 \mid X, \sigma^{2}, \beta\right)\right]\right\}
$$


Donde:

Di: Es la variable binomial (Decisión observada).

$\sigma^{2}$, ß son los parámetros de la distribución normal

$\mathrm{X}$ : son las variables explicativas incorporadas en el modelo

Para el modelo de determinantes de los intentos de suicidio, la alternativa 1 fue haber atentado contra su vida (intento de suicidio), la cual fue la variable dependiente (dicotómica) para este modelo. Las variables independientes utilizadas fueron: edad, estado civil, educación del joven, situación laboral del joven, convivencia con los padres, el maltrato verbal y físico, educación del padre y de la madre, ser víctima de conflicto (desplazado), mala relación con los padres, ser víctima de maltrato físico y verbal, poseer pensamientos suicidas, consumo de alcohol, consumo de drogas y tener conductas irritables. Todas las anteriores variables fueron dicotómicas, excepto la edad.

Los análisis estadísticos se realizaron en el software Stata 13®) (Stata Corporation, College Station, Tx, USA).

\section{Consideraciones éticas}

En la aplicación de la encuesta "Escala de caracterización Ser joven" se siguieron principios éticos de confidencialidad y anonimato, así como la firma del asentimiento y consentimiento informado. Para preservar el anonimato de los encuestados no se utilizaron sus nombres en el análisis de la información.

\section{RESULTADOS}

De una muestra de 10.677 jóvenes de 10-24 años de edad, el $2 \%$ reportó intentos de suicidio. El promedio de edad de los jóvenes que intentaron atentar contra su vida fue de 17,2 años (DE $\pm 3,7)$. El 70,8\% de los intentos de suicidios ocurrió en mujeres. El municipio con mayor frecuencia de intentos de suicidio fue Zambrano y los que registraron menos intentos fueron Calamar y el Carmen de Bolívar (ver Tabla 1). 
Tabla 1.

Prevalencia de intentos de suicidios en adolescentes de siete municipios de los Montes de María.

\begin{tabular}{|c|c|c|c|c|c|c|c|c|c|}
\hline \multirow{2}{*}{ Municipio } & \multicolumn{3}{|c|}{ Femenino } & \multicolumn{3}{|c|}{ Masculino } & \multicolumn{3}{|c|}{ Total } \\
\hline & $\mathrm{N}^{*}$ & ** & $\%$ & $\mathbf{N}^{*}$ & ** & $\%$ & $\mathbf{N}^{*}$ & ** & $\%$ \\
\hline Calamar & 5 & 498 & 1,0 & - & 326 & - & 5 & 824 & 0,6 \\
\hline El Carmen de Bolívar & 14 & 2.151 & 0,7 & 7 & 1.581 & 0,4 & 21 & 3.732 & 0,6 \\
\hline El Guamo & 14 & 463 & 3,0 & 8 & 479 & 1,7 & 22 & 942 & 2,3 \\
\hline San Jacinto & 24 & 750 & 3,2 & 6 & 632 & 0,9 & 30 & 1.382 & 2,2 \\
\hline San Juan Nepomuceno & 22 & 1.044 & 2,1 & 11 & 846 & 1,3 & 33 & 1.890 & 1,7 \\
\hline San Onofre & 26 & 775 & 3,4 & 10 & 513 & 1,9 & 36 & 1.288 & 2,8 \\
\hline Zambrano & 45 & 325 & 13,8 & 20 & 294 & 6,8 & 65 & 619 & 10,5 \\
\hline TOTAL & 150 & 6.006 & 2,5 & 62 & 4.671 & 1,3 & 212 & 10.677 & 2,0 \\
\hline
\end{tabular}

*Frecuencia de intentos de suicidios en adolescentes de siete municipios de los Montes de María.

**Población Encuestada.

Fuente: Cálculos propios a partir de la escala de caracterización Ser Joven.

Según la edad, los intentos de suicidios fueron más frecuentes en menores de 18 años, especialmente en las mujeres. El grupo de edad con mayores intentos fue el de jóvenes entre 16 y 18 años, representando aproximadamente el 30\% del total.

Tabla 2.

Prevalencia de intentos de suicidios en adolescentes por grupo de edad y sexo.

\begin{tabular}{|c|c|c|c|}
\hline Grupo de edad & Sexo & Intentos de suicidio & $\%$ \\
\hline \multirow{3}{*}{10 a 12} & Hombre & 7 & 36,8 \\
\hline & Mujer & 12 & 63,2 \\
\hline & Total & 19 & 9,0 \\
\hline \multirow{3}{*}{13 a 15} & Hombre & 11 & 20,8 \\
\hline & Mujer & 42 & 79,2 \\
\hline & Total & 53 & 25,0 \\
\hline \multirow{3}{*}{16 a 18} & Hombre & 18 & 29,0 \\
\hline & Mujer & 44 & 71,0 \\
\hline & Total & 62 & 29,2 \\
\hline
\end{tabular}




\section{Tabla 2.}

Prevalencia de intentos de suicidios en adolescentes por grupo de edad y sexo.

\begin{tabular}{cccc}
\hline Grupo de edad & Sexo & Intentos de suicidio & $\%$ \\
\hline \multirow{3}{*}{19 a 21} & Hombre & 15 & 36,6 \\
& Mujer & 26 & 63,4 \\
& Total & 41 & 19,3 \\
\hline \multirow{2}{*}{22 a 24} & Hombre & 11 & 29,7 \\
& Mujer & 26 & 70,3 \\
& Total & 37 & 17,5 \\
\hline \multirow{2}{*}{ TOTAL } & Hombre & 62 & 29,2 \\
& Mujer & 150 & 70,8 \\
& Total & 212 & 100 \\
\hline
\end{tabular}

Fuente: Cálculos propios a partir de la escala de caracterización Ser Joven.

Respecto a los intentos de suicidio por grado de escolaridad, la mayoría de los casos ocurrieron en adolescentes que no terminaron el bachillerato. Solo un adolescente sin escolaridad intentó atentar contra su vida. Los jóvenes con nivel de formación técnica presentaron menores intentos de suicidio que los que tenían primaria incompleta y estudios secundarios.

Tabla 3.

Prevalencia de intentos de suicidio en adolescentes por grado de escolaridad.

\begin{tabular}{lc|c}
\hline Escolaridad & Frecuencia Absoluta & $\%$ \\
\hline Sin escolaridad & 1 & 0,5 \\
Primaria completa & 12 & 5,7 \\
Técnico & 12 & 5,7 \\
Primaria incompleta & 23 & 10,9 \\
Secundaria completa & 35 & 16,5 \\
Secundaria incompleta & 129 & 60,9 \\
& & \\
\hline TOTAL & 212 & 100
\end{tabular}

Fuente: Cálculos propios a partir de la escala de caracterización Ser Joven. 
El modelo probit desarrollado para estimar las principales variables que afectan la probabilidad de que un adolescente de los siete municipios estudiados intentara suicidarse, arrojó un pseudo R cuadrado de 0,6537, evidenciando una buena asociación entre las variables regresoras y la regresada. Los jóvenes con pensamientos suicidas ( $(=0,063, p=0,000)$, aquellos que han sido víctimas de abuso físico $(B=0,016, p=0,004)$, y los que tienes padres con bajos niveles de escolaridad ( $\beta=0,014, p=0,004)$ tienen una mayor probabilidad de atentar contra sus vidas que aquellos jóvenes que no presentaron las características antes mencionadas.

Tabla 4.

Modelo probitde los determinantes de los intentos de suicidio en adolescentes de siete municipios de los Montes de María.

\begin{tabular}{|c|c|c|c|c|c|c|}
\hline Variables & $\mathrm{dy} / \mathrm{dx}$ & Std. Err & $\mathbf{z}$ & $\mathrm{P}>$ & \multicolumn{2}{|c|}{ [95\% Conf. Interval] } \\
\hline Edad & 0,0007994 & 0,0007063 & 1,13 & 0,258 & $-0,000585$ & 0,0021838 \\
\hline \multicolumn{7}{|l|}{ Estado civil } \\
\hline Casado(a) & $-0,0210338$ & 0,0067038 & $-3,14$ & 0,002 & $-0,034173$ & $-0,0078946$ \\
\hline Separado(a) & $-0,0089749$ & 0,0091146 & $-0,98$ & 0,325 & $-0,0268392$ & 0,0088894 \\
\hline Unión libre & $-0,004338$ & 0,005179 & $-0,84$ & 0,402 & $-0,0144886$ & 0,0058126 \\
\hline \multicolumn{7}{|l|}{$\begin{array}{l}\text { Escolaridad } \\
\text { del padre }\end{array}$} \\
\hline $\begin{array}{l}\text { Primaria } \\
\text { incompleta }\end{array}$ & 0,0054507 & 0,0054462 & 1 & 0,317 & $-0,0052237$ & 0,0161251 \\
\hline $\begin{array}{l}\text { Primaria } \\
\text { completa }\end{array}$ & $-0,0027081$ & 0,0057196 & $-0,47$ & 0,636 & $-0,0139184$ & 0,0085021 \\
\hline $\begin{array}{l}\text { Secundaria } \\
\text { incompleta }\end{array}$ & 0,01407 & 0,0068367 & 2,06 & 0,04 & 0,0006703 & 0,0274698 \\
\hline $\begin{array}{l}\text { Secundaria } \\
\text { completa }\end{array}$ & 0,0094079 & 0,006835 & 1,38 & 0,169 & $-0,0039884$ & 0,0228042 \\
\hline Universitario & 0,0577642 & 0,0245193 & 2,36 & 0,018 & 0,0097073 & 0,1058211 \\
\hline \multicolumn{7}{|l|}{$\begin{array}{l}\text { Escolaridad } \\
\text { de la madre }\end{array}$} \\
\hline $\begin{array}{l}\text { Primaria } \\
\text { incompleta }\end{array}$ & $-0,0085388$ & 0,0057466 & $-1,49$ & 0,137 & $-0,0198019$ & 0,0027242 \\
\hline $\begin{array}{l}\text { Primaria } \\
\text { completa }\end{array}$ & 0,0017336 & 0,007739 & 0,22 & 0,823 & $-0,0134345$ & 0,0169018 \\
\hline $\begin{array}{l}\text { Secundaria } \\
\text { incompleta }\end{array}$ & 0,0004173 & 0,007076 & 0,06 & 0,953 & $-0,0134513$ & 0,0142859 \\
\hline $\begin{array}{l}\text { Secundaria } \\
\text { completa }\end{array}$ & $-0,0039237$ & 0,0065753 & $-0,6$ & 0,551 & $-0,016811$ & 0,0089635 \\
\hline Universitario & 0,0447664 & 0,033305 & 1,34 & 0,179 & $-0,0205101$ & 0,110043 \\
\hline
\end{tabular}




\section{Tabla 4.}

\section{Modelo probit de los determinantes de los intentos de suicidio en adolescentes de siete municipios de los Montes de María.}

\begin{tabular}{|c|c|c|c|c|c|c|}
\hline $\begin{array}{l}\text { Variables } \\
\text { explicativas }\end{array}$ & $\mathrm{dy} / \mathrm{dx}$ & Std. Err & $\mathrm{z}$ & $P>$ & \multicolumn{2}{|c|}{ [95\% Conf. Interval] } \\
\hline \multicolumn{7}{|l|}{ Empleo } \\
\hline Empleado & $-0,0102739$ & 0,0077389 & $-1,33$ & 0,184 & $-0,0254418$ & 0,0048941 \\
\hline Empleo informal & $-0,0055519$ & 0,0050019 & $-1,11$ & 0,267 & $-0,0153555$ & 0,0042516 \\
\hline Desplazado & $-0,0000905$ & 0,0039548 & $-0,02$ & 0,982 & $-0,0078418$ & 0,0076608 \\
\hline $\begin{array}{l}\text { Mala relación } \\
\text { padres }\end{array}$ & $-0,0047209$ & 0,0096286 & $-0,49$ & 0,624 & $-0,0235926$ & 0,0141509 \\
\hline Abuso sexual & $-0,0114397$ & 0,0144516 & $-0,79$ & 0,429 & $-0,0397642$ & 0,0168848 \\
\hline $\begin{array}{l}\text { Mala relación } \\
\text { hermanos }\end{array}$ & $-0,0027999$ & 0,0074186 & $-0,38$ & 0,706 & $-0,0173402$ & 0,0117403 \\
\hline $\begin{array}{l}\text { Mala relación } \\
\text { padre }\end{array}$ & 0,0056152 & 0,0036342 & 1,55 & 0,122 & $-0,0015077$ & 0,0127381 \\
\hline $\begin{array}{l}\text { Mala relación } \\
\text { madre }\end{array}$ & 0,0032091 & 0,0042635 & 0,75 & 0,452 & $-0,0051473$ & 0,0115654 \\
\hline Maltrato físico & $-0,0167246$ & 0,0057589 & $-2,9$ & 0,004 & $-0,0280119$ & $-0,0054373$ \\
\hline Maltrato verbal & $-0,003164$ & 0,0057371 & $-0,55$ & 0,581 & $-0,0144086$ & 0,0080805 \\
\hline Embarazo & 0,0087334 & 0,0055149 & 1,58 & 0,113 & $-0,0020756$ & 0,0195423 \\
\hline $\begin{array}{l}\text { Consumo } \\
\text { de alcohol }\end{array}$ & 0,005224 & 0,0080257 & 0,65 & 0,515 & $-0,0105062$ & 0,0209541 \\
\hline $\begin{array}{l}\text { Pensamientos } \\
\text { suicidas }\end{array}$ & 0,0629042 & 0,0050201 & 12,53 & 0 & 0,0530649 & 0,0727434 \\
\hline $\begin{array}{l}\text { Conductas } \\
\text { irritables }\end{array}$ & 0,0061228 & 0,0040179 & 1,52 & 0,128 & $-0,0017522$ & 0,0139978 \\
\hline
\end{tabular}

Note: $d y / d x$ for factor levels is the discrete change from the base level.

Fuente: Cálculos propios a partir de la escala de caracterización Ser Joven.

\section{ANÁLISIS Y DISCUSIÓN}

El presente estudio describió y exploró los determinantes de los intentos de suicidio de jóvenes pertenecientes a siete municipios de la subregión de los Montes de María. Ésta, se conforma por 15 municipios de los departamentos de Bolívar y Sucre, con una población total de 596.914 (según el DANE), con la mayoría entre los 10-20 años. La subregión de los Montes de María es principalmente pobre, dado los altos índices de necesidades básicas 
insatisfechas que presentan sus municipios, como son los casos de El Carmen de Bolívar, San Jacinto y Chalán (Ramírez, 2012).

Teniendo en cuenta la región estudiada, y de acuerdo al modelo probit y al análisis descriptivo presentado, se evidenció el nivel de escolaridad del joven y de los padres, las ideaciones suicidas y el abuso o maltrato físico son importantes predictores de los intentos de suicidios en 212 jóvenes de siete municipios de los Montes de María que reportaron haber atentado contra su vida. En nuestro estudio, la mayoría de los intentos de suicidio ocurrieron entre los 16 y 18 años de edad y la minoría entre los 10 y 12 años. Esto coincide con el hecho que los intentos de suicidio son poco frecuentes antes de la pubertad, su incidencia aumenta entre los 12 y 13 años y alcanza un pico entre los 17 y 24 años de edad (Kaplan HI, 2009)(Gómez-Restrepo, Carlos. Rodriguez Malagón, Nelcy. Bohórquez P, 2002).

Resultados similares a los de nuestro estudio, muestran que en México mediante una encuesta de representatividad nacional, las mujeres, las personas que no están casadas o en unión libre y los de menor escolaridad tienen riesgos más elevados de presentar conducta suicida (Borges, Medina-Mora, Orozco, \& Ouéda, 2009).

En nuestro país, un estudio similar desarrollado en Pasto, encontró que la prevalencias de intentos de suicidio estaba alrededor del 15\%, siendo mas de 7 veces que la encontrada en nuestra investigación ( $2.05 \%$ ) , estando estas prevalencias por debajo de las encontradas en estudios en otros países que oscilan entre el 11 y el 25\%, (González-Macip S, Diaz A, Ortiz S, González-Forteza C, 2000)(González-Forteza C, Ramos L, Caballero MA, 2003) en el cual la violencia sexual, el maltrato físico intrafamiliar, y el vivir alejado de la familia fueron los principales determinantes de intentos de suicidio. (Hernán, 2009).

Los datos poblacionales sobre la ocurrencia en los grupos de edades son muy poco frecuentes para estudios de intento de suicidio (Moscicki, 1999)(Gómez Carlos, 2002) limitando así su realización. Para nuestro caso, el tamaño de la muestra es lo suficientemente grande que permite identificar eventos poco frecuentes, como es el caso de los intentos de suicidio.

El presente estudio presenta limitaciones. Se destaca el potencial error de medida del cuestionario "escala de caracterización ser Joven" utilizado como principal insumo para desarrollar este trabajo. El error de medida se presenta en todos los estudios y se da principalmente por la captura, en algunos casos inadecuada, de las respuestas por el instrumento (Rada, 2004). En el caso de nuestro estudio, el error de medida proviene de dos factores involucrados en el proceso de recolección de la información, tales como, errores generados por los entrevistados y errores propios del cuestionario. En el primero, se destaca la ausencia de conocimiento sobre los aspectos que son preguntados, la poca comprensión de las preguntas del cuestionario o las apreciaciones acerca de la temática de la investigación, dada la baja escolaridad de la población encuestada. En el segundo, el cuestionario registró preguntas objetivas escritas como preguntas de respuestas subjetivas. Estos elementos son potenciales sesgos de los resultados descritos en nuestro estudio. Sin embargo, a pesar de estas limitaciones, los resultados del estudio constituyen una evidencia importante de la situación actual referente al perfil de jóvenes vulnerables con intentos suicidas de siete municipios de los Montes de María. Además, la muestra fue lo suficientemente grande para establecer cierto grado de representatividad y no encontrar diferencias significativas dentro de los grupos de análisis.

En definitiva, explorar las características de jóvenes que intentaron suicidarse, permite generar mecanismos de acción por parte de los tomadores de decisiones que eviten nuevas 
manifestaciones suicidas, especialmente en una región de país tradicionalmente afectada por fenómenos como el desplazamiento, la pobreza y la guerra. Además, el presente estudio es una invitación a seguir analizando las características sociodemográficas y sanitarias de los jóvenes de esta región del país.

\section{REFERENCIAS BIBLIOGRÁFICAS}

Borges, G., Medina-Mora, M., Orozco, R., \& Ouéda, C. (2009). Distribución y determinantes sociodemográficos de la conducta suicida en México. Salud Mental.

Diana Carolina Aguirre-Florez, Jose Jaime Castaño-Castrillon, F. M.-S. et al. (2013). Riesgo suicida y factores asociados en adolescentes en tres colegios de la ciudad de Manizales (Colombia). Revista de Facultad de Medicina, 63(3), 419-29. http://doi.org/http://dc.doi.org/10.15446/ revfacmed.v63n.44205

Durkheim, E. (1995). El suicidio. Coyoacán, Mexico.

Gómez Carlos, et al. (2002). Factores asociados al intento de suicidio en la población colombiana. Revista Colombiana de Psiquiatría, 271-286.

Gómez-Restrepo, Carlos. Rodriguez Malagón, Nelcy. Bohórquez P, A. et al. (2002). Factores Asociados al intento de suicido en la población colombiana. Revista Colombiana de Psiquiatría, 31(4).

González-Forteza C, Ramos L, Caballero MA, W. F. (2003). Correlatos psicosociales de depresión, ideación e intento suicida en adolescentes mexicanos. Psicothema, 15, 524-532.

González-Macip S, Diaz A, Ortiz S, González-Forteza C, G.-N. J. (2000). Características psicométricas de la EScala de Ideación Suicida de Beck (ISB) en estudiantes universitarios de la ciudad de México. Salud Mental, 23, 21-30.

Gutierez Ana, Contreras Carlos, O. R. (2011). El suicidio, conceptos actuales. Revista Unimar, 27-84. Retrieved from http://www.redalyc.org/pdf/582/58229510.pdf

Hernán, V.-G. F. (2009). Situación de la conducta suicida en estudiantes de colegios y universidades de San Juan de Pasto, Colombia. Salud Mental, 32(2). Retrieved from http://www.scielo. org.mx/scielo.php?pid=S0185-33252009000200009\&script=sci_arttext INS. (2015).

Kaplan HI, S. (2009). Comprehensive textbook of psychiatry (9th ed.). Philadelphia.

Moscicki, E. (1999). Identification of suicide risk factors using epidemiologic studies. Psychiatr Clin Norht Am, 20(4), 499-517.

OMS. (2014). La prevención de suicidio. Luxemburgo: Organizacion Mundial de la Salud.

OMS. (2015). Retrieved from http://www.who.int/mediacentre/factsheets/fs351/es/

Pérez Isabel, et al. Factores asociados al intento suicida e ideación suicida persistente en un centro de atención primaria. (2004). Bogotá.

Rada, V.D. de. (2004). Problemas de representatividad en las encuestas con muestreos probabilísticos. Papers 74, 45-66.

Ramírez, J. (2012). Caracterización socio-demográfica del área de desarrollo rural de los montes de maría. Retrieved from http://www.incoder.gov.co/documentos/Estrategia de Desarrollo Rural/Pertiles Territoriales/ADR Montes de Maria/Perfil Territorial/CARACTERIZACION SOCIO-DEMOGRAFICA MONTES DE MAR\%C3\%8DA.pdf 